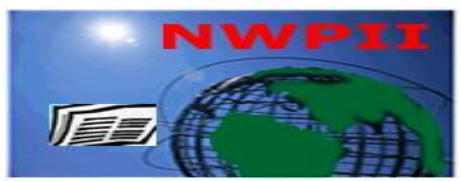

\title{
Carbon Fiber Belly Board Quality Assurance in Radiotherapy
}

\author{
Aime M. Gloi, Javis Jackson, Angela Sonnier
}

GenesisCare,1316 Nelson Avenue, Modesto CA 95350,USA

*Corresponding Author

Aime M. Gloi

GenesisCare

1316 Nelson Avenue, Modesto CA 95350

USA

Phone: 209-575-5870

Fax: 209-575-5870

Email: agloi7288@gmail.com

Received:14 May 2021; | Revised:26 May 2021; | Accepted:06 September 2021

\begin{abstract}
Background: Carbon fiber belly board is the newer immobilization device that has earned recognition in sparing small bowel during prone pelvic irradiation. It is characterized by mechanical strength, a smooth rigidity, and a low specific density. In this study, the beam attenuation for a $10 \times 10 \mathrm{~cm}^{2}$ field size was calculated for 6 and $18 \mathrm{MV}$ photons beam.

Methods: A Farmer ion chamber of $0.6 \mathrm{cc}$ volume, slabs of solid water phantom, and an electrometer were used to measure the relative exit dose with or without the carbon fiber belly board at variable gantry angles. The measurements were performed at $100 \mathrm{~cm} \mathrm{SSD}$, and $100 \mathrm{MU}$ were delivered.

Results: The average beam attenuation amounted to $3.46 \pm 1.53 \%$ and $2.22 \pm 0.89 \%$ for 6 and $18 \mathrm{MV}$, respectively. The highest attenuation was obtained at $130^{\circ}$ and the lowest at $120^{\circ}$ gantry angle for both energies.

Conclusion: Irradiating through carbon fiber immobilization devices triggered a variation of the beam attenuation at different gantry angles. As a result, there is a loss of skin-sparing effect with megavoltage photon beam. Therefore, special attention is needed in the treatment planning system when using these carbon fiber immobilization devices.
\end{abstract}

Keywords: Carbon Fiber, Quality assurance, Beam attenuation

\section{Introduction}

Treatments setup and reproducibility are essential in radiotherapy. The use of immobilization devices ensures the stability of the patient's position during and subsequent treatments. Although these devices are important, they are made of material that attenuates the incident beam, thus interfering with the tumor dose coverage, and altering the dose distribution. Furthermore, there is ample evidence that using carbon fiber immobilization devices results in the loss of the skin-sparing effect.

Several authors ${ }^{[1-6]}$ have investigated different aspects of the radiation beam attenuation and 
surface dose of the linear accelerator (linac) couch top, chiefly made of carbon fiber by assessing its characteristics. They do not cause any computed tomographic (CT) artifacts and exhibited a high mechanical strength. Further, they showed a high beam transmission and a low specific density, leading to its widespread application in immobilization devices.

The belly board considered in this study, is made of carbon fiber. It is primarily used to decrease the volume of the irradiated small bowel during prone pelvic radiotherapy. It allows for the small bowel to shift and constrict because of its configuration. Its impact has become paramount mainly in the volumetric modulated arc therapy (VMAT) treatments. However, it is patientdependent due to the physical body load variability. Thus, patient position will be affected, leading to a trade-off with bowel toxicity. Allal et al., [7] revealed in previous studies, that a combination of a belly board with the prone position is susceptible to setup errors. They found an increased reproducibility displacement in the anteriorposterior direction with a belly board compared to no belly board in the prone position. In addition, several reports ${ }^{[8-9]}$ have shown the carbon fiber immobilization devices to be another source of beam attenuation coupled with the couch top. They concluded that a failure to consider these inherent factors will result in skin or target dose change.

To date and our knowledge, no study was undertaken to investigate the carbon fiber belly board beam attenuation. The report aimed to elucidate the carbon fiber belly board attenuation at various gantry angles for a $10 \times 10 \mathrm{~cm}^{2}$ field size using 6 and $18 \mathrm{MV}$ photon beams.

\section{Materials and Methods}

All measurements were acquired through a Varian 21 EX (Varian Medical Systems, Palo Alto, CA) dual photons energies (6 and $18 \mathrm{MV}$ ) using a $30 \times 30 \times 30 \mathrm{~cm}^{3}$ solid water phantom placed at a $100 \mathrm{~cm}$ source-to-surface distance (SSD). The data were collected via an Accredited Dosimetry Calibration Laboratory (ADCL) calibrated Farmertype chamber Exradin A12 and an electrometer A MAX4000 (Standard Imaging, Madison, WI). The measurements were performed with and without the carbon fiber belly board (CIVCO, Orange City, IA, USA) at variable gantry angles starting at 180 degrees with 10 degrees increments to 120 degrees. The carbon fiber belly board components whose goal is to decrease the irradiated small bowel volume during prone pelvic radiotherapy are illustrated in Figure (1). It is composed of a caudal aperture for the pubic bone position while the central portion aid in the forward compression of the small bowel towards the major aperture. Also, the rooted fiducial crosshair is used as a secondary setup reference. Figures $(2 \mathrm{a}, \mathrm{b})$ displays the experimental setup for the beam attenuation measurement through the carbon fiber belly board and solid water slabs. The ionization chamber was placed at the isocenter with $10 \times 10 \mathrm{~cm}^{2}$ field size and exposed to $100 \mathrm{MU}$. The resulting percentage attenuation was then calculated as:

Attenuation $=\left(1-\mathrm{D}_{\mathrm{c}} / \mathrm{D}_{\mathrm{nc}}\right) * 100 \%(1)$

where Dc represents the charges measured with the beam passing through the carbon fiber belly board and Dnc represents the charges measured without the carbon fiber belly board.

\section{Results}

A beam attenuation of carbon fiber belly board was measured for 18 and 6MV photon energy at $10 \times 10 \mathrm{~cm} 2$ field size. A graphical representation of the frontal and sagittal view of a patient undergoing volumetric modulated arc therapy (VMAT) pelvis irradiation using the carbon fiber belly board is shown in Figures $(3 a, b)$. The data were enabled by using an ionization chamber through a combination of belly board and water slabs. The magnitude of the beam attenuation measured by the ionization chamber through the couch and the combination of the couch and belly board is expressed in Figure (4), depicting the charges collected as the gantry angle varies. Table 1 summarized the calculated attenuation for both energies. The magnitude attenuation of $18 \mathrm{MV}$ photons beams was lower compared to $6 \mathrm{MV}$. In fact, it was $3.467 \pm 1.538 \%$ and $2.225 \pm 0.894 \%$ for 6 and $18 \mathrm{MV}$, respectively at $10 \times 10 \mathrm{~cm}^{2}$ field size.

Figures (5a, b, and c) showed the angular distribution of the carbon fiber belly board attenuation for both energies. As estimated, all measurements show higher attenuation values as the 
gantry angles increases. This is in part due to the beam obliquity as the path length through the couch, belly board and water slabs increases. The highest beam attenuation was detected at $130^{\circ}$ gantry angle for both energies yielding an attenuation of 5.77\% and $3.67 \%$ for 6 and $18 \mathrm{MV}$, respectively. The least attenuation was observed at $120^{\circ}$ gantry angle with $1.15 \%, 1.41 \%$ for 6 and $18 \mathrm{MV}$, respectively.

\section{Discussion}

The objective of this study was to perform quality assurance for a new immobilization device. Due to their abundance and widespread application, it is incumbent to check for the material integrity, strength, durability, and beam attenuation for the treatment planning system (TPS) where different materials densities have been configured. One of the most vital benefits of the megavoltage beam in radiotherapy is its skin-sparing effect. Couch attenuation coupled with immobilization devices made of carbon fiber supplants the buildup region and lessens the skin-sparing effect resulting in an increased skin reaction. ${ }^{[10]}$

In this report, the calculated beam attenuation is in agreement with that of the manufacturer: $2.8 \%$ vs. $3.467 \pm 1.538 \%$, (our measurement) for $6 \mathrm{MV}$ photon beams. The results revealed higher attenuation at $6 \mathrm{MV}$ compared to $18 \mathrm{MV}$ due to beam hardening and emphasized the bolus effect. Besides, the results demonstrated that the attenuation rises with increasing beam obliquity and is angular dependent [11]. Anjanappa et al., [12] reported on the benefits of using the belly board in terms of reducing the setup error and acute small bowel toxicity. Mok et al., ${ }^{[13]}$ echoed the same sentiment by highlighting the improvement in the clinically relevant doses to the small bowel in the treatment of rectal carcinoma. Indeed, several reports supported prone position using belly board for pelvic radiation. ${ }^{[14-16]}$

While these events may influence the dose distribution, they are not presently taken into account in some commercially available TPSs nor the full impact of these immobilization devices have been elucidated. The reliance on manufacturer account should be examined by a quality assurance. It is reasonable to apply the characteristics of welldiscussed carbon fiber couch tops to the carbon fiber belly board.

\section{Conclusion}

We have investigated the beam attenuation through the carbon fiber belly board as a function of photon energy and gantry rotation. The obtained results showed a more pronounced beam attenuation at $130^{\circ}$ angle incidence for both energies regardless of belly board or not. These attenuation measurements might be a valuable verification tool to substantiate the manufacturer's claims to be considered in the treatment planning system.

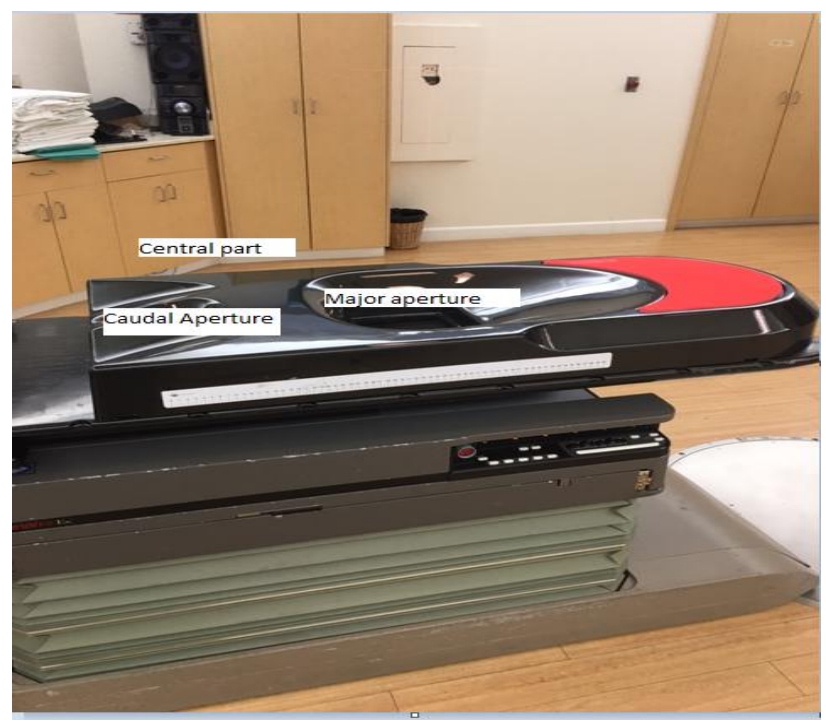

Figure 1: Illustration of the carbon fiber belly board used in prone pelvic irradiation 


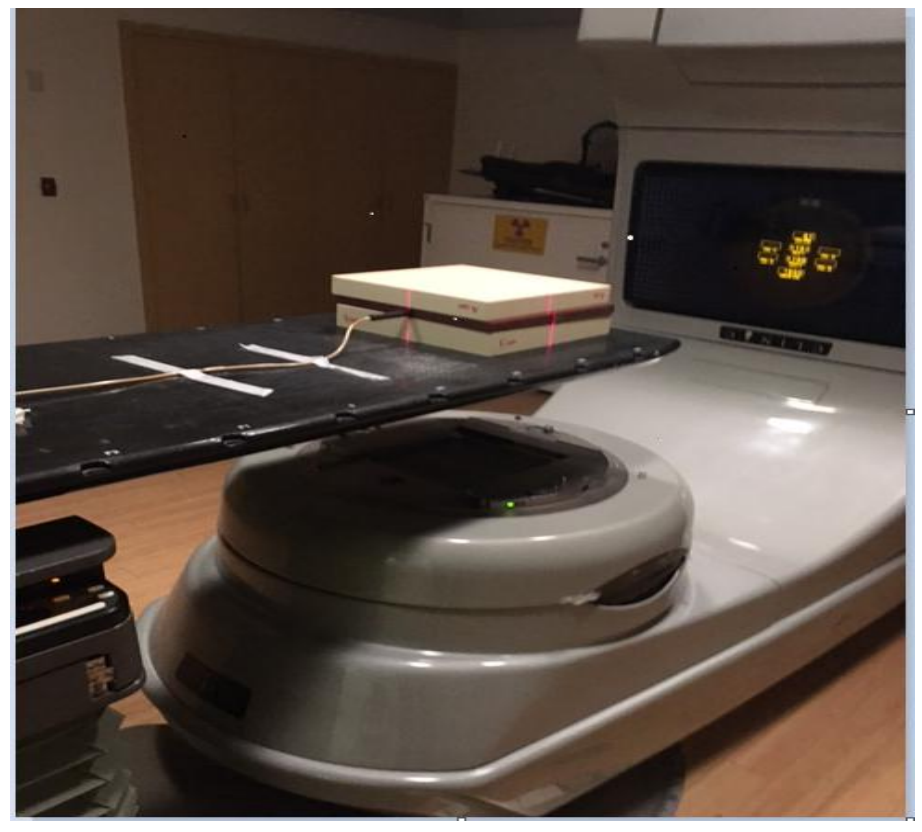

Figure 2a: Setup for the beam attenuation measurement as a function of photon energy and gantry rotation through $10 \mathrm{~cm}$ solid water slab

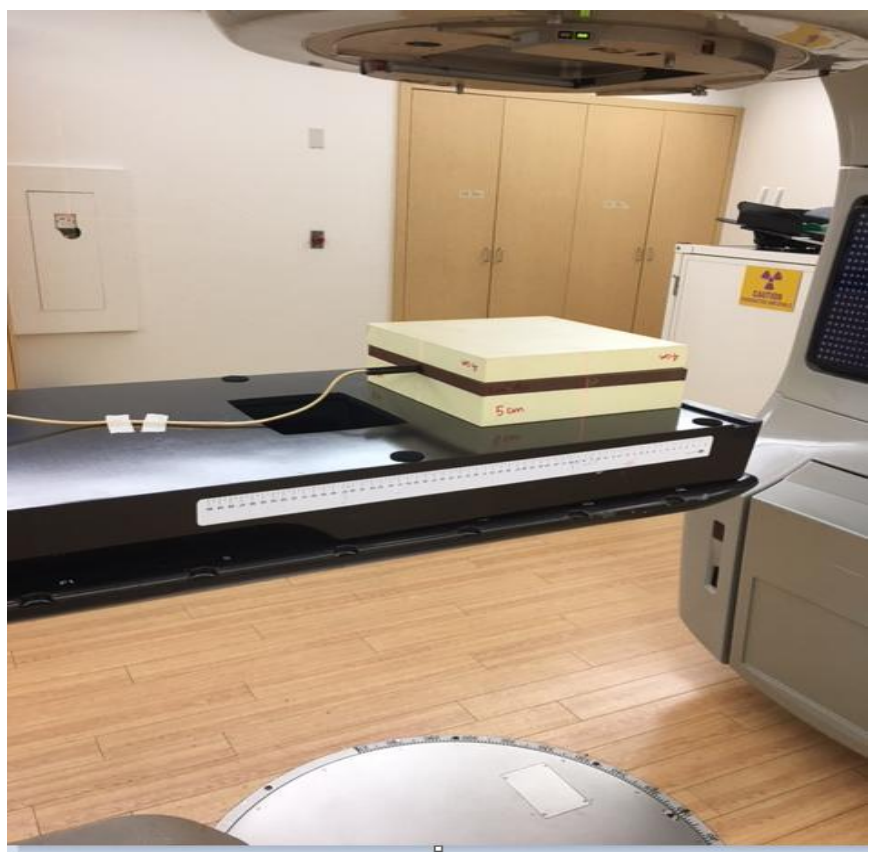

Figure 2b: Setup for Setup for the beam attenuation measurement as a function of photon energy and gantry rotation through $10 \mathrm{~cm}$ solid water slab and a carbon-fiber belly board 


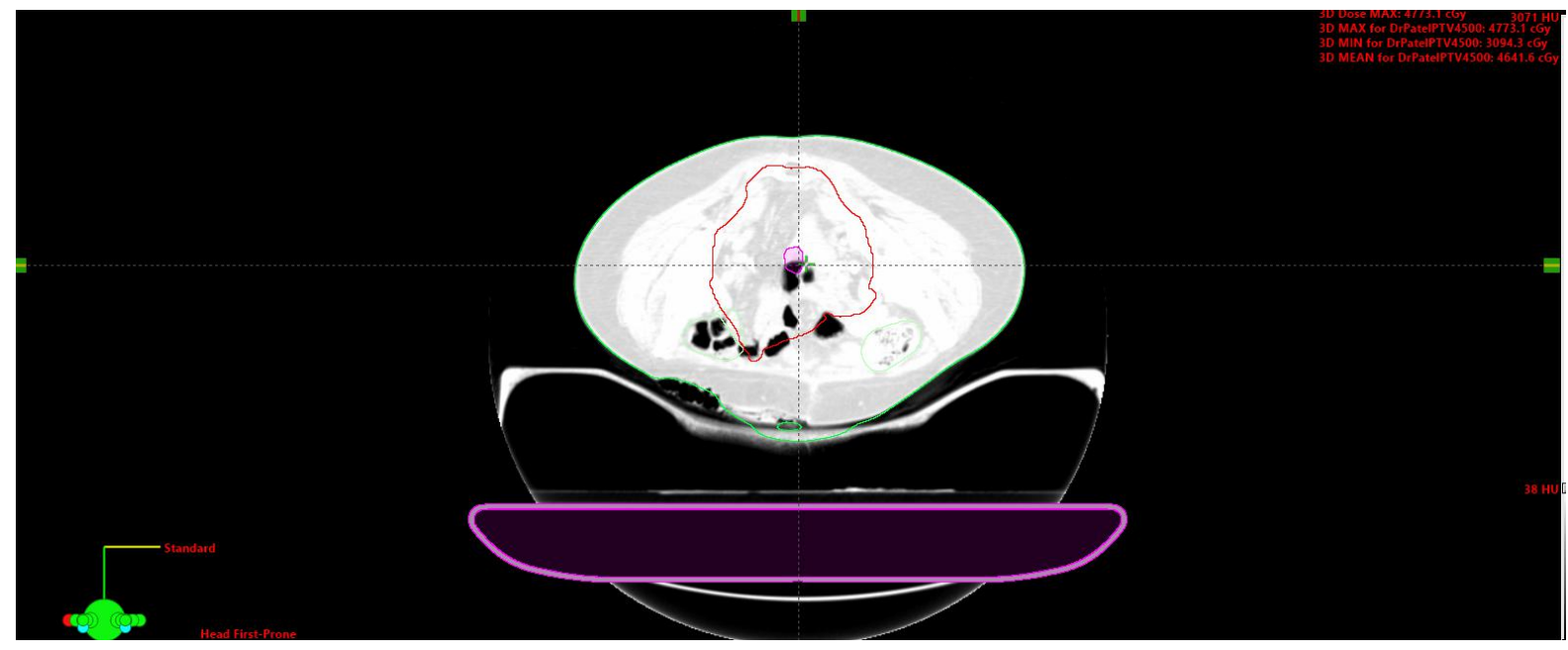

Figure 3a: Frontal view of a patient undergoing VMAT pelvis irradiation using Carbon Fiber belly board $(180 \times 25=4500 c G y)$

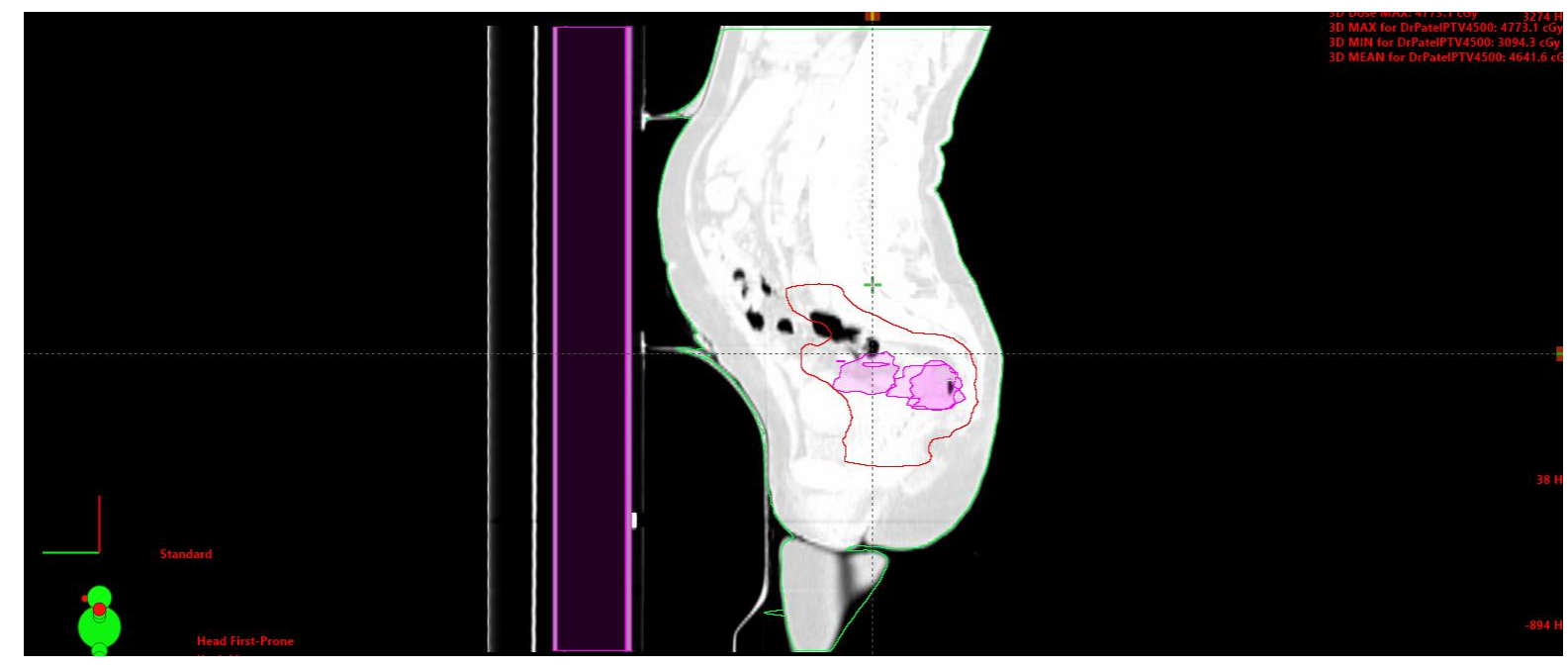

Figure 3b: Sagittal view of a patient undergoing VMAT pelvis irradiation using Carbon Fiber belly board $(180 \times 25=4500 c G y)$

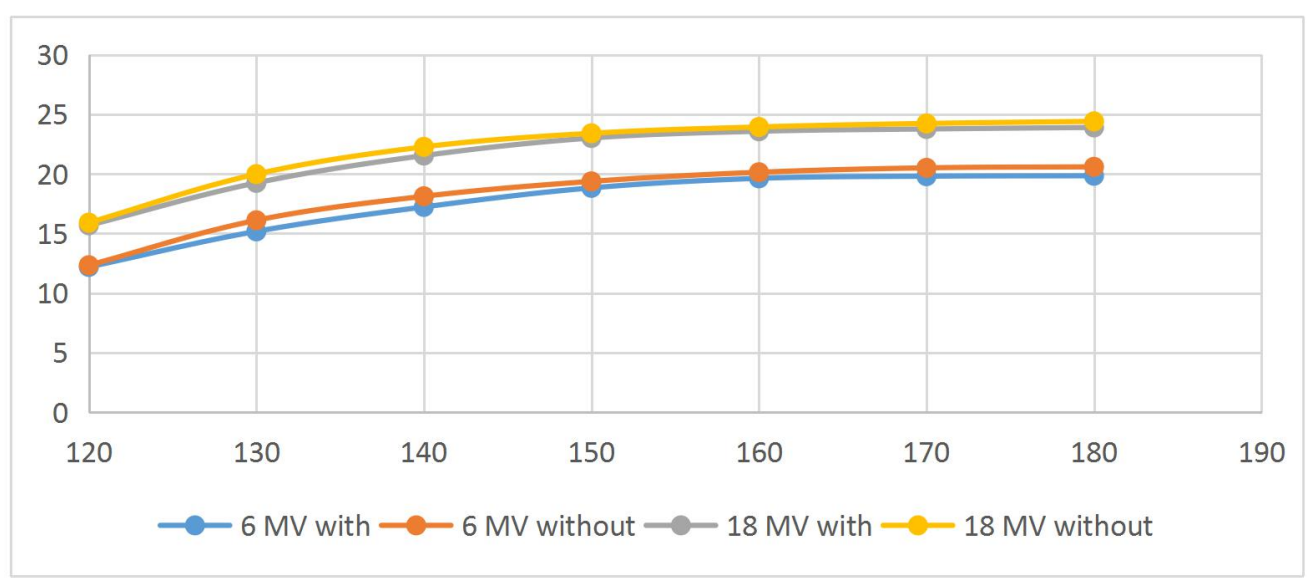

Figure 4: Charges collected during angular distribution for $6 \mathrm{MV}$ and $18 \mathrm{MV}$ for $10 \times 10 \mathrm{~cm}^{2}$ field size. The $\mathrm{x}$ axis denote gantry angle in degree and $y$-axis charged collected in $\mathrm{nC}$ 


\section{Attenuation at $6 \mathrm{MV}$}

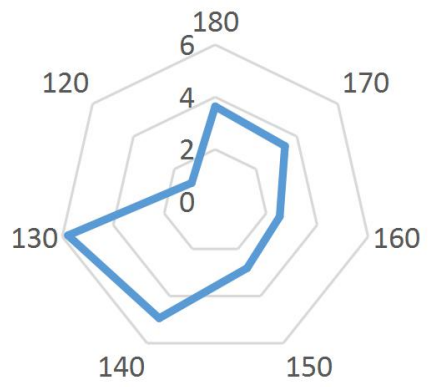

Figures 5a: Angular distribution of attenuation for $6 \mathrm{MV}$ photon beam

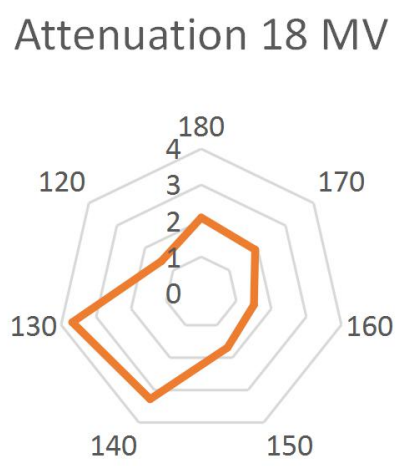

Figures 5b: Angular distribution of attenuation for $18 \mathrm{MV}$ photon beam

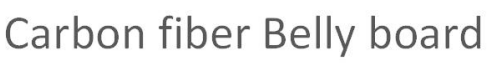

$\longrightarrow$ Att6 $=$ Att18

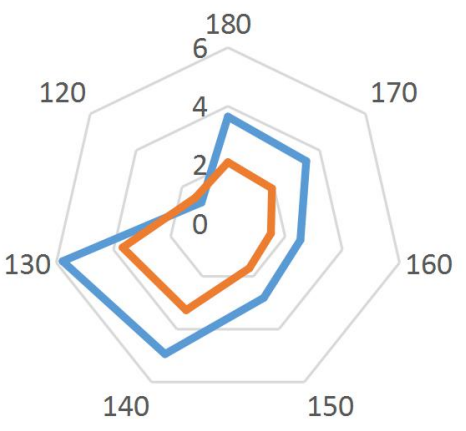

Figures 5c: Angular distribution of attenuation for both $6 \mathrm{MV}$ and $18 \mathrm{MV}$ photon beam 
Table 1: Calculated beam attenuation using an ionization chamber for 6 and $18 \mathrm{MV}$ at variable gantry angles with $100 \mathrm{MU}$ and a field size of $10 \times 10 \mathrm{~cm}^{2}$

\begin{tabular}{|l|l|l|}
\hline \multirow{2}{*}{ Angle degrees } & \multicolumn{2}{c|}{ Attenuation (\%) } \\
\cline { 2 - 3 } & $6 \mathrm{MV}$ & $18 \mathrm{MV}$ \\
\hline 180 & 3.643 & 2.090 \\
\hline 170 & 3.414 & 1.919 \\
\hline 160 & 2.533 & 1.540 \\
\hline 150 & 2.814 & 1.688 \\
\hline 140 & 4.940 & 3.279 \\
\hline 130 & 5.776 & 3.679 \\
\hline 120 & 1.151 & 1.414 \\
\hline Average & 3.467 & 2.225 \\
\hline Std & 1.538 & 0.894 \\
\hline
\end{tabular}

\section{References}

1 Silva C,Mateus D,Vieira S,Rodrigues M,Eiras M, Greco C (2016). Radiotherapy couches: is Kevlar an obstacle? Attenuation study of three different tabletops. Journal of radiotherapy in Practice 15:346-353. doi.org/10.1017/S1460396916000297

2 Vieira SC, Kaatee RS, Dirkx ML, Heijmen BJ. Two-dimensional measurement of photon beam attenuation by the treatment couch and immobilization devices using an electronic portal imaging device. Med Phys 2003; 30(11): 2981-2987 DOI: $10.1118 / 1.1620491$

3 Poppe B, Chofor N, Ruhmann A, Kunth W, Djouguela A, Kollhoff R, Willborn KC. The effect of a carbon-fiber couch on the depthdose curves and transmission properties for megavoltage photon beams. Strahlenther Onkol 2007; 183(1): 43-48 [PMID: 17225945 DOI: $10.1007 / \mathrm{s} 00066-007-1582-8]$

4 McCormack S, Diffey J, Morgan A. The effect of gantry angle on megavoltage photon beam attenuation by a carbon fiber couch insert. Med
Phys 2005; 32(2): 483-487 [PMID: 15789595 DOI: $10.1118 / 1.1852792]$

5 Gerig LH, Niedbala M, Nyiri BJ. Dose perturbations by two carbon fiber treatment couches and the ability of a commercial treatment planning system to predict these effects. Med Phys 2010; 37(1): 322-328 [PMID: 20175495 DOI: 10.1118/1.3271364]

6 Njeh CF, Raines TW, Saunders MW. Determination of the photon beam attenuation by the Brainlab imaging couch: angular and field size dependence. J Appl Clin Med Phys 2009; 10(3): 16-27 DOI: 10.1120/jacmp.v10i3.2979

7 Allal AS, Bischof S, Nouet P. Impact of the "belly board" device on treatment reproducibility in preoperative radiotherapy for rectal cancer. Strahlenther Onkol 2002; 178(5): 259-262 DOI: $10.1007 / \mathrm{s} 00066-002-$ $\underline{0889-8}$

8 Mihaylov IB, Bzdusek K, Kaus M. Carbon fiber couch effects on skin dose for volumetric modulated arcs. Med Phys 2011; 38(5): 24192423 DOI: $\underline{10.1118 / 1.3576106}$ 
9 Vanetti E, Nicolini G, Clivio A, Fogliata A, Cozzi L. The impact of treatment couch modelling on RapidArc. Phys Med Biol 2009; 54(9): N157-166 [PMID: 19351984 DOI: 10.1088/0031-9155/54/9/N03]

10 Lee $\mathrm{KW}, \mathrm{Wu} \mathrm{JK}$, Jeng SC, Hsueh Liu YW, Cheng JC. Skin dose impact from vacuum immobilization device and carbon fiber couch in intensity modulated radiation therapy for prostate cancer. Med Dosim 2009; 34(3): 228232 DOI: $10.1016 /$ j.meddos.2008.10.001

11 Li H, Lee AK, Johnson JL, Zhu RX, Kudchadker RJ. Characterization of dose impact on IMRT and VMAT from couch attenuation for two Varian couches. J Appl Clin Med Phys 2011; 12(3): 3471 DOI: $\underline{\text { 10.1120/jacmp.v12i3.3471 }}$

12 Anjanappa M, Rajeev KR, James FV, Kumar A, Mathews S, Bhaskaran S, Vasudevannair SS, Govindan JP: The impact of patient positioning and use of belly board on small bowel toxicity in patients receiving pelvic radiotherapy for gynecological malignancies. Journal of cancer research and therapeutics, 2017, Vol.13, issue3 pp519-523 doi.org/10.16/j.adro.2017.01.005
13 Mok $\mathrm{H}$, Crane $\mathrm{CH}$, Palmer $\mathrm{MB}$ intensity modulated radiation therapy (IMRT): differences in target volumes and improvement in clinically relevant doses to small bowel in rectal carcinoma. Radiat Oncol 6, 63 (2011). doi.org/10.1186/1748-717X-6-63

14 Martin J, Fitzpatrick K, Horan G, McCloy R, Buckney S, O'Neill L, Faul C. Treatment with a belly-board device significantly reduces the volume of small bowel irradiated and results in low acute toxicity in adjuvant radiotherapy for gynecologic cancer: results of a prospective study. Radiother Oncol 2005; 74(3): 267-274 DOI: $\underline{10.1016 / \text { j.radonc.2004.11.010 }}$

15 Pinkawa M , Gagel B , Demirel C,Schmachtenberg A, Asadpour B, Eble MJ. Dose- volume histogram evaluation of prone and supine patient position in external beam radiotherapy for cervical and endometrial cancer. Radiother Oncol 2003; 69:99- 105. Doi: 10.1016/s0167-8140(03)00244-5

16 Adli M, Mayr NA, Kaiser HS, Skwarchuk MW, Meeks SL, Mardirossian G, et al. Does prone positioning reduce small bowel dose in pelvic radiation with intensity- modulated radiotherapy for gynecologic cancer? Int $\boldsymbol{J}$ Radiat Oncol Biol Phys 2003; 57:230- 8. Doi: 10.1016/s0360-3016(03)00409-7 